\title{
UK Food Standards Agency $\alpha$-linolenic acid workshop report
}

\author{
Peter Sanderson $^{1}$, Yvonne E. Finnegan ${ }^{2}$, Christine M. Williams ${ }^{2}$, Philip C. Calder ${ }^{3}$, \\ Graham C. Burdge ${ }^{3}$, Stephen A. Wootton ${ }^{3}$, Bruce A. Griffin ${ }^{4}$, D. Joe Millward ${ }^{4}$, Nicholas C. Pegge ${ }^{5}$ and \\ Wanda J. E. Bemelmans ${ }^{6}$ \\ ${ }^{1}$ Nutrition Division, Food Standards Agency, Aviation House, 125 Kingsway, London WC2 6NH, UK \\ ${ }^{2}$ Hugh Sinclair Unit of Human Nutrition, School of Food Biosciences, University of Reading, Whiteknights, PO Box 226, \\ Reading RG6 6AP, UK \\ ${ }^{3}$ Institute of Human Nutrition, School of Medicine, University of Southampton, Southampton SO16 7PX, UK \\ ${ }^{4}$ Centre for Nutrition and Food Safety, School of Biomedical and Life Sciences, University of Surrey, \\ Guildford GU2 7XH, UK \\ ${ }^{5}$ Department of Pharmacology, Therapeutics and Toxicology, University of Wales College of Medicine, \\ Cardiff CF14 $4 X N$, UK \\ ${ }^{6}$ University of Groningen, Department of General Practice, Anton Deusinglaan 4, 9713 AW, Groningen, The Netherlands
}

(Received 7 June 2002 - Accepted 17 June 2002)

\begin{abstract}
The UK Food Standards Agency convened a group of expert scientists to review current research investigating whether $n-3$ polyunsaturated fatty acids (PUFA) from plant oils ( $\alpha$-linolenic acid; ALA) were as beneficial to cardiovascular health as the $n$-3 PUFA from the marine oils, eicosapentaenoic acid (EPA) and docosahexaenoic acid (DHA). The workshop also aimed to establish priorities for future research. Dietary intake of ALA has been associated with a beneficial effect on CHD; however, the results from studies investigating the effects of ALA supplementation on CHD risk factors have proved equivocal. The studies presented as part of the present workshop suggested little, if any, benefit of ALA, relative to linoleic acid, on risk factors for cardiovascular disease; the effects observed with fish-oil supplementation were not replicated by ALA supplementation. There is a need, therefore, to first prove the efficacy of ALA supplementation on cardiovascular disease, before further investigating effects on cardiovascular risk factors. The workshop considered that a beneficial effect of ALA on the secondary prevention of CHD still needed to be established, and there was no reason to look further at existing CHD risk factors in relation to ALA supplementation. The workshop also highlighted the possibility of feeding livestock ALA-rich oils to provide a means of increasing the dietary intake in human consumers of EPA and DHA.
\end{abstract}

$\alpha$-Linolenic acid: Fish oils: Cardiovascular disease: Nutrition research

The Food Standards Agency (FSA) convened a workshop on 18 March 2002, to examine the role of $\alpha$-linolenic acid (ALA) in relation to cardiovascular disease. The results from recently completed studies were presented, both FSA- and non-FSA-funded, and the workshop was chaired by Professor Martijn Katan, Wageningen University, The Netherlands. The aim of the workshop was to determine where this work has taken us and where further work should be concentrated, as well as acting as a vehicle for dissemination. The research recommendations emanating from the workshop discussions will feed into the future direction of the UK FSA-funded nutrition research, and may also be of value in guiding other funders internationally.

\section{Background}

The long chain $n-3$ polyunsaturated fatty acids (PUFA), eicosapentanoic acid (EPA; 20:5n-3) and docosahexaenoic

\footnotetext{
Abbreviations: ALA, $\alpha$-linolenic acid; apo, apolipoprotein; BBSRC-LINK, Biotechnology and Biological Sciences Research Council-LINK; DHA, docosahexaenoic acid; DPA, docosapentaenoic acid; EPA, eicosapentaenoic acid; FSA, Food Standards Agency; LA, linoleic acid; MARGARIN, Mediterranean Alpha-linolenic Enriched Groningen Dietary Intervention; PC, phosphatidylcholine; PL, phospholipid; PUFA, polyunsaturated fatty acids; TAG, triacylglycerol.

* Corresponding author: Dr Peter Sanderson, fax +44 207276 8906, email peter.sanderson@foodstandards.gsi.gov.uk
} 
acid (DHA; $22: 6 n-3$ ), have been shown to reduce the reoccurrence of CHD (GISSI-Prevenzione Investigators, 1999). EPA and DHA also have a variety of beneficial effects on risk factors for CHD. Oily fish is the richest dietary source of EPA and DHA, but fish consumption is low in habitual UK diets (Department of the Environment, Food and Rural Affairs, 2001). An alternative source of $n-3$ PUFA is the more abundant ALA (18:3n-3), which can be elongated and desaturated to its long-chain derivatives EPA and DHA; however, in man the extent and regulation of this conversion is unclear. The question arises, therefore, whether plant oils rich in ALA (for example, linseed, rapeseed and nut oils) could reproduce the beneficial effects of fish consumption on risk for cardiovascular disease, via conversion of ALA to its long-chain derivatives.

The Lyon Diet Heart Study (de Lorgeril et al. 1994, 1999) suggested that dietary ALA may have a remarkably beneficial effect on the secondary prevention of CHD; however, compared with the control diet, the experimental diet also had a higher content of fruit and vegetables, legumes, fibre-rich cereals, monounsaturated fatty acids, less linoleic acid (LA; $18: 2 n-6)$ and less saturated fatty acids, making it impossible to dissect out the role of individual food components. Several prospective epidemiological studies have reported a protective effect of ALArich foods against CHD (Dolecek, 1992; Ascherio et al. 1996; Hu et al. 1999), although a more recent study of elderly men (Oomen et al. 2001) found no association between ALA intake and CHD.

The results from studies investigating the effects of ALA supplementation on CHD risk factors have been equivocal. ALA has been shown to be equivalent to LA and $n$-6-rich oils for lipid and lipoprotein effects (Harris, 1997; Pang et al. 1998) and, unlike fish-oil supplementation, results in no effect on plasma triacylglycerol (TAG) levels (Pang et al. 1998). The overall results from studies investigating any effect of ALA on haemostasis have proved inconclusive (Knapp, 1997; Allman-Farinelli et al. 1999), whereas fish-oil supplementation can lead to positive effects on haemostasis (Mutanen \& Freese, 2001). Arterial endothelial function, a non-invasive marker of arterial health, can be improved by fish-oil supplementation (Nestel, 2000), and one study has reported a beneficial effect of a high intake of ALA $(20 \mathrm{~g} / \mathrm{d})$ on endothelial function in obese subjects; however, insulin sensitivity and HDL-cholesterol diminished concomitantly (Nestel et al. 1997). Inflammation is a characteristic of many diseases, including CHD. Fish-oil supplementation has been shown to exhibit inhibitory effects on most tests of immune function and on inflammatory markers in human subjects (Calder, 2001). There are only a few studies of the immune and inflammatory effects of ALA in human subjects. These show that supplementation with high intakes of ALA (14 and $18 \mathrm{~g} / \mathrm{d}$ ) can partly inhibit lymphocyte proliferation and the production of inflammatory cytokines (Kelley et al. 1991; Caughey et al. 1996). Studies supplementing with lower amounts of ALA ( 2 or $4 \mathrm{~g} / \mathrm{d})$ reveal limited impact (Healy et al. 2000; Thies et al. 2001a,b,c).

The studies presented as part of the present workshop investigate the importance of ALA as a source of long chain $n-3$ PUFA and its influence on risk factors for
CHD. These studies further the understanding of the effects of ALA intake on haemostasis, endothelial function, immune function, lipoprotein metabolism and its conversion to long-chain derivatives.

The effects of plant- and marine-derived $n-3$ polyunsaturated fatty acids on coronary heart disease risk factors in moderately hyperlipidaemic subjects

Dr Yvonne Finnegan presented the findings from a fourcentre Department for Environment, Food and Rural Affairs-Biotechnology and Biological Sciences Research Council-LINK (BBSRC-LINK) collaborative study cosponsored by Unilever and Hoffmann-la Roche and coordinated at the University of Reading. The aim was to evaluate the relative effects of dietary EPA plus DHA compared with estimated biologically equivalent amounts of dietary ALA on a range of risk factors for CHD. Following a 4week run-in period during which all subjects consumed a typical $n$-6- PUFA rich margarine (control), 150 moderately hyperlipidaemic men and women (aged 25-72 years) were randomised into one of five diets enriched in either EPA+DHA (to a total daily intake of $0.8 \mathrm{~g}, n-6: n-3$ ratio 9.6 ; or $1.7 \mathrm{~g} / \mathrm{d}, n-6: n-3$ ratio 6.5$)$ or to biologically equivalent amounts of ALA (to a total daily intake of $4.5 \mathrm{~g}, n-6: n-3$ ratio 3.3 ; or $9.5 \mathrm{~g} / \mathrm{d}, n-6: n-3$ ratio 1.3 ) or to control (no additional $n-3$ PUFA, $n-6: n-3$ ratio $10 \cdot 5$ ) for a period of 6 months. The $n-3$ PUFA were incorporated in the form of a $25 \mathrm{~g}$ portion of fat spread to replace the subject's normal margarine or spread and three oil capsules. The main findings from the study were as follows.

There was no significant effect of any of the $n-3$ interventions compared with the control on fasting serum lipids (total, LDL- and HDL-cholesterol, TAG, non-esterified fatty acids), glucose or insulin or on the postprandial TAG or glucose response following 6 months dietary intervention. There was a trend for total and LDL-cholesterol levels to be increased following the $1.7 \mathrm{~g}$ EPA+DHA/d intervention, which was shown to be significant for total cholesterol in females on EPA+DHA $(+17.4$ (SD 3.9) \%) compared with the control females ( +4.0 (SD 2.5); $P<0 \cdot 01)$.

Retrospective genotyping for apolipoprotein (apo) E polymorphisms of subjects was carried out since previous work in our group had shown subjects with an apo E4 genotype to be more responsive to the cholesterol-raising effects of EPA and DHA (Minihane et al. 2000). In the present study, a trend towards an increase in LDL-C in the apo E4 subjects was evident with a $7.1 \%$ decrease, $10.3 \%$ increase and $19.9 \%$ increase in LDL-C in the apo E2, E3 and E4 groups respectively following the $1.7 \mathrm{~g}$ $\mathrm{EPA}+\mathrm{DHA} / \mathrm{d}$ diet. However the small number of subjects precludes definitive conclusions from this retrospective analysis. An important question in this study was whether ALA (at biologically equivalent levels) can mimic the TAG-lowering effects of EPA+DHA. The findings suggest no TAG-lowering effects of ALA and indicate the reverse may be true since the mean change in fasting TAG following an intake of $9.5 \mathrm{~g} \mathrm{ALA} / \mathrm{d}$ was $+10.9 \%$ compared with a decrease $(-7.7 \%)$ in the EPA+DHA $(1.7 \mathrm{~g} / \mathrm{d})$ group $(P<0 \cdot 05)$. 
There was no significant effect of any of the $n-3$ interventions on systolic or diastolic blood pressure, on markers of the blood coagulation (factor VIIc, factor VIIag, factor VIIa, factor XIIa) or fibrinolytic (tissue plasminogen activator, plasminogen activator inhibitor) systems, or on markers of innate or acquired immune function compared with control. An additional in vivo functional measure of immune function (delayed hypersensitivity reaction) also showed no effect of any of the $n-3$ interventions.

There was an increase in the susceptibility of ex vivo LDL to oxidation following an intake of $1.7 \mathrm{~g}$ EPA+DHA/d. Dietary ALA did not affect the susceptibility of ex vivo LDL to oxidation. There was no effect of any of the $n-3$ interventions on markers of antioxidant status (plasma $\alpha$-tocopherol, ferric-reducing ability of plasma) measured in this study.

As expected both the EPA+DHA diets led to increases in the proportion of EPA and DHA in plasma phospholipids (PL) $(P<0 \cdot 05)$. Both ALA diets also led to significant enrichment of plasma PL with ALA and EPA $(P<0.05)$. However, there was no change in the proportion of DHA in the plasma PL despite the long-term supplementation with ALA. The change in the proportion of plasma PL EPA following the ALA diets was comparable to that observed following the $1.7 \mathrm{~g}$ EPA+DHA/d diet suggesting that the ratio of 7:1 we had used in estimating biologically equivalent levels of dietary ALA and EPA+DHA was approximately true. There was no incorporation of ALA in platelet or mononuclear cell PL, although the proportion of EPA was significantly increased following the higher ALA $(9.5 \mathrm{~g} / \mathrm{d})$ diet $(P<0 \cdot 05)$. However, consistent with plasma PL, no significant changes in the proportion of DHA in cell membrane PL were observed following ALA supplementation.

In conclusion: (i) in middle-aged male and females, increasing ALA in the diet increases tissue and circulating EPA but not DHA levels; (ii) at feasible dietary levels (5$10 \mathrm{~g} / \mathrm{d}$ ), ALA does not appear to mimic the reported effects of the long-chain $n$-3 PUFA on blood lipids, haemostatic factors or immune and inflammatory function; (iii) apart from modest reductions in plasma TAG, previously reported beneficial effects of EPA+DHA on blood lipids, haemostatic and immune and inflammatory function were not observed in the present study and may reflect the lower levels of EPA and DHA used; (iv) potentially adverse effects of EPA and DHA on total and LDL-cholesterol may be modulated by gender and genotype.

\section{The effects of altered meal and dietary $n-3$ polyunsaturated fatty acid intakes upon conversion of $\alpha$-linolenic acid to longer-chain fatty acids (in men)}

Dr Steve Wootton presented results from studies designed to characterise ALA conversion in men using stable-isotope technology either following a test meal with differing n-3 fatty acid content (FSA-funded) or after increasing dietary consumption of ALA or EPA and DHA (as part of the BBSRC-LINK study).

The first phase of studies investigated the acute effect of meal $n-3$ fatty acid content upon ALA conversion. Six men consumed one of three test meals on each of three occasions: control (1 g ALA, n-3:n-6 ratio 0.15); high ALA (6.3 g, $n-3: n-6$ ratio 0.64); high EPA+DHA (0.77g, $n-3: n-6$ ratio $0 \cdot 28)$. Following the consumption of the control meal, ALA was mobilised from the gut as chylomicron TAG, while conversion to longer-chain fatty acids appeared to occur primarily in the liver and entered the circulation esterified to phosphatidylcholine (PC) probably on VLDL. The relative excursions of labelled $n-3$ fatty acids in total plasma lipids over $21 \mathrm{~d}$ were ALA $84 \%$, EPA $7.9 \%$ and docosapentaenoic acid (22:5n-3, DPA) $8.1 \%$. There was no significant enrichment of DHA in any lipid class. Incorporation of labelled ALA, EPA and DPA into erythrocyte $\mathrm{PC}$, followed a similar time-course to plasma PC which suggests exchange of intact PC between lipoproteins and erythrocyte membranes. No significant effects of meal $n-3$ fatty-acid content were observed upon the conversion, or fractional oxidation, of $\left[{ }^{13} \mathrm{C}\right] \mathrm{ALA}$.

The second phase of studies examined the chronic effect of altering the background diet $n-3$ fatty-acid content on ALA conversion. The extent of conversion of ALA to longer-chain fatty acids was determined over $48 \mathrm{~h}$ in a sub-group of men ( $n$ 14) participating in the BBSRCLINK study, reported earlier. At baseline, the relative cumulative excursions of labelled fatty acids in total plasma were ALA $95.6 \%$, EPA $2.8 \%$, DPA $1.2 \%$ and DHA $0.04 \%$. The lower fractional enrichments in EPA and DPA reflect the shorter time period over which measurements were made. Increased dietary ALA (substrate) did not drive an increase in ALA conversion, but increasing dietary EPA+DHA (product) exerted a significant product inhibition on EPA and DPA synthesis from ALA. DHA synthesis was unaffected, suggesting that hepatic DHA synthesis may be regulated independently from EPA and DPA. There was no significant effect of $n-3$ fatty acid intake on the partitioning of $\left[{ }^{13} \mathrm{C}\right] \mathrm{ALA}$ to oxidation. In agreement with previous studies (Salem et al. 1999; Vermunt et al. 2000; Pawlosky et al. 2001), these results indicate that conversion of ALA to EPA is limited and to DHA, at best, marginal in well-nourished individuals. It remains to be determined whether this process can be up regulated during periods of increased demands for $n$-3 long-chain PUFA.

Preliminary data from studies of ALA conversion in women were also presented, suggesting that in 20-30year-olds ALA can be converted to DHA (9\%) to a greater extent than in men. It was suggested that this may be hormonally up regulated, which raises the possibility that DHA synthesis may increase in pregnancy and lactation to meet the demands of the fetus and neonate for DHA. Whether this observation has direct implications for risk of cardiovascular disease is unclear.

\section{Importance of $\alpha$-linolenic acid as a source of long-chain $n-3$ polyunsaturated fatty acid and its influence on risk factors for cardiovascular disease}

Dr Bruce Griffin and Professor Joe Millward presented results from an FSA-funded study investigating the relative effects of dietary ALA and fish oil on risk factors for cardiovascular disease. In collaboration with Dr Steve Wootton, this study also examined the effect of the 
background diet ALA:LA ratio on ALA conversion to its long-chain derivatives using stable-isotope technology. Sixty male subjects with an atherogenic lipoprotein phenotype (TAG $>1.5 \mathrm{mmol} / \mathrm{l}$, HDL $<1 \mathrm{mmol}$ and a predominance of small, dense LDL) were recruited to a 12-week dietary intervention trial. The subjects received one of three diets: control (n-6:n-3 ratio 30); ALA (15 g/d, n6: $n-3$ ratio 0.46); EPA+DHA (3 g/d, $n-6: n-3$ ratio 5). The diets were modified using sachets of oil, cooking oils, margarines and capsules. The main findings from the study were as follows.

The fish-oil diet increased erythrocyte EPA (2.5 to $6.6 \%$ ) and DHA (4.7 to $7.8 \%)$, whereas the high ALA diet increased ALA $(0.3$ to $1.2 \%)$ and EPA $(1.3$ to $3.2 \%)$, but not DHA (4.0 to $4.3 \%$ ). All diets decreased serum cholesterol levels; serum TAG levels were decreased by the fish-oil diet $(-0.59 \mathrm{mmol} / \mathrm{l}, P<0.001)$ and the ALA diet $(-18 \%, \mathrm{NS})$; though there were no significant differences between diets on serum cholesterol, TAG or in HDL- or LDL-cholesterol.

LDL subclasses showed a significant redistribution towards larger, less dense particles only after fish oil, with a fall in the relative abundance of small, dense LDL (density $1.040-1.060 \mathrm{~g} / \mathrm{ml}) \quad(P<0.001)$. There was no change in measures of haemostatic function including factor VII, plasminogen activator inhibitor-1 and fibrinogen in response to any diet, or flow-mediated dilatation after cuff-induced hyperaemia (endothelium-dependent) or glyceryl trinitrate-induced vasodilatation (endotheliumindependent) in a subset of subjects on each diet.

In all subjects, conversion of $\left[{ }^{13} \mathrm{C}\right] \mathrm{ALA}$ to $\left[{ }^{13} \mathrm{C}\right] \mathrm{EPA}$ and $\left[{ }^{13} \mathrm{C}\right] \mathrm{DPA}$ isolated from both erythrocyte and plasma PC fractions has been observed with very low (but significant) levels of $\left[{ }^{13} \mathrm{C}\right] \mathrm{DHA}$ formation. The $14 \mathrm{~d}{ }^{13} \mathrm{C}$ distribution between ALA, EPA, DPA and DHA was 22, 53, 21 and $4 \%$ respectively (mean values for erythrocyte and plasma PC on both diets). The higher proportion of label in EPA than in ALA (not theoretically possible for a unidirectional, true precursor-product relationship) may partly reflect an underestimation of the ALA labelling if peak labelling of ALA occurred before the $24 \mathrm{~h}$ blood sample, or may indicate differential exchange rates between plasma ALA and EPA and intra-hepatic pools. The effect of background diets on $\left[{ }^{13} \mathrm{C}\right] \mathrm{ALA}$ conversion rates involved calculation of standardised ${ }^{13} \mathrm{C}$ values for EPA, DPA and DHA to correct for the varying precursor enrichment and this indicated that mean values for $\left[{ }^{13} \mathrm{C}\right] \mathrm{EPA}$ were higher after the flax diet, suggestive of higher conversion, but not significantly so given the marked between-subject variation. A much larger group, and considerable expense, would be required to demonstrate any significant difference.

The fish-oil diet produced predictably favourable changes in blood lipids and lipoproteins, but had no impact on the measures of haemostatic and endothelial function that have been associated with the high risk dyslipidaemia in these subjects. The high-ALA diet produced mild effects on serum lipids in relation to the fish-oil diet, but produced no other measurable changes in our endpoints. The isotopic and membrane fatty-acid data indicate that ALA was rapidly converted to EPA, and to DPA to a significantly lesser degree, but is only very slowly converted to DHA, at least as indicated by sampling of blood-lipid pools. It may be that this low rate of DHA synthesis is adequate to provide for usual metabolic demands in these subjects. However it cannot increase DHA to the level achieved by dietary DHA from fish oil thus preventing any fish-oil-like effects.

\section{A double-blind controlled trial of dietary enrichment with $\alpha$-linolenic acid on vascular endothelial function in human subjects}

Dr Nicholas Pegge presented results from an FSA-funded randomised controlled dietary intervention trial examining the effect of supplementation with ALA in a healthy, freeliving population. The principal outcome measure was assessment of arterial endothelial function. Secondary outcome measures included measurement of blood lipids and lipoproteins, homocysteine, vitamin status, full blood counts, clotting factors, and measures of oxidative stress. Two hundred healthy volunteers were supplemented for 6 months with one of four dietary interventions: capsules of ALA ( $2 \mathrm{~g} / \mathrm{d})$; placebo capsules; bread milled with flax seed sufficient to yield an equivalent amount of ALA to capsule supplementation; placebo bread. The main findings from the study were as follows.

Both forms of active supplementation increased the concentration of ALA and EPA, but not DHA, in plasma relative to placebo, but to a greater extent in those taking active capsules than flax seed-enriched bread (62 v. $18 \%$; $P<0.001$ and $P=0.01$ respectively; $P=0.001$ for the difference).

Supplementation with pure encapsulated ALA decreased arterial pulse wave velocity responsiveness to reactive hyperaemia, indicative of a detrimental effect on endothelial function (reduced from 5.0 to $2.4 \%$ in the arm, and from 8.2 to $4.9 \%$ in the leg; $P=0.055$ and $P<0.001)$. Neither active or placebo bread nor placebo capsules had any significant effect on endothelial function.

ALA capsules decreased the plasma concentrations of ApoA1 and ApoB (from 1.31 to $1.23, P=0.007$ and from $1 \cdot 16$ to $1 \cdot 06, P=0 \cdot 049)$, but no intervention had any significant effect on macronutrient intake, fasting lipid, homocysteine, vitamin $\mathrm{A}, \mathrm{C}$ or $\mathrm{E}$ levels, or full blood counts.

This study suggests that there is no benefit to vascular health from eating foods enriched with ALA, or taking supplements of ALA, which may indeed be detrimental.

\section{Effect of an increased intake of $\alpha$-linolenic acid on cardiovascular risk factors: the Mediterranean Alpha-linolenic Enriched Groningen Dietary Intervention study}

The aim of the Mediterranean Alpha-linolenic Enriched Groningen Dietary Intervention (MARGARIN) study was to investigate the effects of an increased intake of ALA on cardiovascular risk factors in a high-risk population (Bemelmans et al. 2002). The study was double-blind and participants were randomly assigned to receive either an LA-rich margarine (58\% LA, $0.3 \%$ ALA; $n$ 157) or an ALA-enriched margarine (46\% LA, $15 \%$ ALA; $n$ 109) over 2 years. The subjects were hypercholesterolaemic 
(6-8 $\mathrm{mmol} / \mathrm{l})$ and had at least two other cardiovascular risk factors (117 men, 149 women, $55 \pm 10$ years). Among the exclusion criteria were diabetes mellitus, and the use of cholesterol-lowering drugs and acetylsalicylic acid. The main findings from the study were as follows.

Among ALA-users, the contents of ALA and EPA in the cholesteryl ester increased, and the content of arachidonic acid, $n-6: n-3$ ratio and LA:ALA ratio decreased as compared with the LA-users $(P<0 \cdot 01)$.

ALA-users had a higher total cholesterol:HDL-cholesterol ratio (net difference $+0.34 ; 95 \%$ CI $0.12,0.56$ ), lower HDL-cholesterol level $(-0.05 \mathrm{mmol} / \mathrm{l} ; 95 \% \mathrm{CI}$ $-0 \cdot 10,0)$, higher TAG level $(+0.24 \mathrm{mmol} / \mathrm{l} ; 95 \% \mathrm{CI}$ 0.02 , 0.46), lower plasma fibrinogen level $(-0.18 \mathrm{~g} / \mathrm{l}$; $95 \%$ CI $-0.31,-0.04$; after 1 year), and lower C-reactive protein level $(-21 \mathrm{mg} / \mathrm{l} ; 95 \% \mathrm{CI}-4 \cdot 3,0)$ than the LAusers.

No significant differences existed in intima-media thickening of the carotid and femoral arteries, auto-antibodies against oxidised LDL, von Willebrand factor, and soluble intercellular adhesion molecule.

In this study increased ALA intake had unfavourable effects on the serum lipid profile, but had favourable effects on markers for inflammation (C-reactive protein, fibrinogen) when investigated against an LA-rich background diet. In the longer term, the anti-inflammatory effects may outweigh the adverse effects on serum lipids, with regard to cardiovascular events; however, due to the short follow-up period and insufficient power of the MARGARIN study it was impossible to investigate this. Future research in primary prevention should focus on the events as an outcome and on exploring a potential anti-inflammatory mechanism.

\section{Discussion}

In adults, ALA supplementation causes an increase in the blood and plasma levels of ALA, EPA and DPA but hardly any, if at all, DHA. Increasing DHA in the diet increases blood and plasma DHA levels. It was noted that DHA precursor $n-3$ PUFA could be transported via the plasma to tissues where subsequent conversion might occur (for example, myocytes, astrocytes), and further isotopic studies need to investigate other compartments as well as blood.

The studies presented as part of the present workshop suggest little, if any, benefit of ALA, relative to LA, on risk factors for cardiovascular disease; the effects observed with fish-oil supplementation were not replicated by ALA supplementation. No consistent beneficial effects of ALA supplementation were observed on measures of endothelial function, immune function, serum lipids or measures of haemostatic function; whereas the long-chain derivatives of ALA (EPA and DHA) have been demonstrated to have beneficial effects. Although ALA supplementation increases EPA there is no demonstrable benefit on the risk factors investigated. There is a need, therefore, to first prove the efficacy of ALA supplementation on cardiovascular disease, before further investigating effects on cardiovascular risk factors.

The main cause of sudden death from acute heart attack is ventricular fibrillation. Both clinical and animal studies have suggested that $n$-3 PUFA have anti-arrhythmic effects (Christensen et al. 1999, 2000; Leaf, 1999); indeed, the most likely mechanism for the beneficial effect of EPA and DHA on the re-occurrence of CHD is the stabilisation of arrhythmias (Marchioli et al. 2002). It has been postulated that this could be an underlying mechanism for the beneficial effect of the Mediterranean ALA-rich diet in the secondary prevention of CHD (de Lorgeril et al. 1994, 1999), either directly or via conversion to EPA (Connor, 1999; Leaf, 1999). The present workshop, however, had reservations about the evidence suggesting a beneficial effect of ALA on the secondary prevention of CHD, and felt this still needed to be established.

The present workshop also highlighted the possibility of feeding livestock ALA-rich oils to provide a means of increasing the dietary intake in human consumers of EPA and DHA. Issues such as the higher costs incurred to producers would have to be addressed, as well as the need to provide industry with clear scientific advice.

\section{Recommendations}

1. For further isotopic research, measures of the different pools are needed (for example, heart), as well as cheaper isotopes.

2. There is no reason to look further at existing CHD risk factors in relation to ALA supplementation.

3. For a randomised clinical trial to investigate the effect of ALA supplementation on the re-occurrence of CHD.

\section{Participants}

Professor Martijn Katan, Wageningen University, The Netherlands; Dr Yvonne Finnegan, University of Reading; Dr Sam Kew, University of Reading; Dr Bruce Griffin, University of Surrey; Dr Steve Wootton, University of Southampton; Dr Nicholas Pegge, University of Wales College of Medicine, Cardiff; Dr Wanda Bemelmans, University of Groningen, The Netherlands; Dr William Gilmore, Northern Ireland Centre for Diet and Health, University of Ulster; Professor Christine Williams, University of Reading; Dr Anne Marie Minihane, University of Reading; Professor Tom Sanders, King's College, London; Professor Joe Millward, University of Surrey; Dr Philip Calder, University of Southampton; Dr Susan Jebb, Human Nutrition Research, Cambridge; Dr Carmel Moore, Human Nutrition Research, Cambridge; Professor Rudolph Riemersma, University of Edinburgh; Professor Klaus Wahle, Rowett Research Institute, Aberdeen; Dr Mark Ramsey, University of Wales College of Medicine, Cardiff; Dr Graham Burdge, University of Southampton; Dr Tim Key, Cancer Research UK Epidemiology Unit, Oxford; Dr Christina Goodacre, Department for Environment, Food and Rural Affairs; Dr Judy Buttriss, British Nutrition Foundation; Ms Nilani Sritharan, Nutrition Society; Professor Ian Givens, ADAS; Dr Kevin Shingfield, ADAS, University of Reading; Ms Anne Heughan, Unilever and Food and Drink Federation; Dr Elke Trautwein, Unilever Research Vlaardingen; Dr Ray Rice, 
International Society for the Study of Fatty Acids and Lipids; Dr Reto Muggli, Hoffmann-La Roche; Mr René Josse, Hoffmann-La Roche; Dr Lisa Jackson, FSA; Ms Alette Weaver, FSA; Ms Rachel Elsom, FSA; Dr Peter Sanderson, FSA.

\section{References}

Allman-Farinelli MA, Hall D, Kingham K, Pang D, Petocz P \& Favaloro EJ (1999) Comparison of the effects of two low fat diets with different $\alpha$-linolenic:linoleic acid ratios on coagulation and fibrinolysis. Atherosclerosis 142, 159-168.

Ascherio A, Rimm EB, Giovannucci EL, Spiegelman D, Stampfer M \& Willett WC (1996) Dietary fat and risk of coronary heart disease in men: cohort follow up study in the United States. British Journal of Medicine 313, 84-90.

Bemelmans WJ, Broer J, Feskens EJ, Smit AJ, Muskiet FA, Lefrandt JD, Bom VJ, May JF \& Meyboom-de Jong B (2002) Effect of an increased intake of alpha-linolenic acid and group nutritional education on cardiovascular risk factors: the Mediterranean Alpha-linolenic Enriched Groningen Dietary Intervention (MARGARIN) study. American Journal of Clinical Nutrition 75, 221-227.

Calder PC (2001) N-3 polyunsaturated fatty acids, inflammation and immunity: pouring oil on troubled waters or another fishy tale? Nutrition Research 21, 309-341.

Caughey GE, Mantzioris E, Gibson RA, Cleland LG \& James MJ (1996) The effect on human tumor necrosis factor alpha and interleukin 1 beta production of diets enriched in n-3 fatty acids from vegetable oil or fish oil. American Journal of Clinical Nutrition 63, 116-122.

Christensen JH, Christensen MS, Dyerberg J \& Schmidt EB (1999) Heart rate variability and fatty acid content of blood cell membranes: a dose-response study with n-3 fatty acids. American Journal of Clinical Nutrition 70, 331-337.

Christensen JH, Christensen MS, Toft E, Dyerberg J \& Schmidt EB (2000) Alpha-linolenic acid and heart rate variability. Nutritional Metabolism and Cardiovascular Disease 10, $57-61$.

Connor WE (1999) Alpha-linolenic acid in health and disease. American Journal of Clinical Nutrition 69, 827-828.

de Lorgeril M, Renaud S, Mamelle N, Salen P, Martin JL, Monjaud I, Guidollet J, Touboul P \& Delaye J (1994) Mediterranean alpha-linolenic acid-rich diet in secondary prevention of coronary heart disease. Lancet 343, 1454-1459.

de Lorgeril M, Salen P, Martin JL, Monjaud I, Delaye J \& Mamelle N (1999) Mediterranean diet, traditional risk factors, and the rate of cardiovascular complications after myocardial infarction: final report of the Lyon Diet Heart Study. Circulation 99, 779-785.

Department of the Environment, Food and Rural Affairs (2001) The National Food Survey. London: H.M. Stationery Office.

Dolecek TA (1992) Epidemiological evidence of relationships between dietary polyunsaturated fatty acids and mortality in the multiple risk factor intervention trial. Proceedings of the Society for Experimental Biology and Medicine 200, 177-182.

GISSI-Prevenzione Investigators (1999) Dietary supplementation with n-3 polyunsaturated fatty acids and vitamin E after myocardial infarction: results of the GISSI-Prevenzione trial. Gruppo Italiano per lo Studio della Sopravvivenza nell'Infarto miocardico. Lancet 354, 447-455.

Harris WS (1997) n-3 fatty acids and serum lipoproteins: human studies. American Journal of Clinical Nutrition 65, 1645S$1654 \mathrm{~S}$.

Healy DA, Wallace FA, Miles EA, Calder PC \& Newsholme P
(2000) Effect of low-to-moderate amounts of dietary fish oil on neutrophil lipid composition and function. Lipids 35, $763-768$.

Hu FB, Stampfer MJ, Manson JE, Rimm EB, Wolk A, Colditz GA, Hennekens CH \& Willett WC (1999) Dietary intake of alpha-linolenic acid and risk of fatal ischemic heart disease among women. American Journal of Clinical Nutrition 69, 890-897.

Kelley DS, Branch LB, Love JE, Taylor PC, Rivera YM \& Iacono JM (1991) Dietary alpha-linolenic acid and immunocompetence in humans. American Journal of Clinical Nutrition 53, 40-46.

Knapp HR (1997) Dietary fatty acids in human thrombosis and hemostasis. American Journal of Clinical Nutrition 65, $1687 \mathrm{~S}-1698 \mathrm{~S}$.

Leaf A (1999) Dietary prevention of coronary heart disease: the Lyon Diet Heart Study. Circulation 99, 733-735.

Marchioli R, Barzi F, Bomba E, Chieffo C, Di Gregorio D, Di Mascio R, Franzosi MG, Geraci E, Levantesi G, Maggioni AP, Mantini L, Marfisi RM, Mastrogiuseppe G, Mininni N, Nicolosi GL, Santini M, Schweiger C, Tavazzi L, Tognoni G, Tucci C \& Valagussa F (2002) Early protection against sudden death by n-3 polyunsaturated fatty acids after myocardial infarction: time-course analysis of the results of the Gruppo Italiano per lo Studio della Sopravvivenza nell'Infarto Miocardico (GISSI)-Prevenzione. Circulation 105, 1897-1903.

Minihane AM, Khan S, Leigh-Firbank EC, Talmud PJ, Wright JW, Murphy MC, Griffin BA \& Williams CM (2000) ApoE polymorphism and fish oil supplementation in subjects with an atherogenic lipoprotein phenotype. Arteriosclerosis, Thrombosis and Vascular Biology 20, 1990-1997.

Mutanen M \& Freese R (2001) Fats, lipids and blood coagulation. Current Opinion in Lipidology 12, 25-29.

Nestel PJ (2000) Fish oil and cardiovascular disease: lipids and arterial function. American Journal of Clinical Nutrition 71, 228S-231S.

Nestel PJ, Pomeroy SE, Sasahara T, Yamashita T, Liang YL, Dart AM, Jennings GL, Abbey M \& Cameron JD (1997) Arterial compliance in obese subjects is improved with dietary plant n-3 fatty acid from flaxseed oil despite increased LDL oxidizability. Arteriosclerosis, Thrombosis and Vascular Biology 17, 1163-1170.

Oomen CM, Ocke MC, Feskens EJ, Kok FJ \& Kromhout D (2001) alpha-Linolenic acid intake is not beneficially associated with $10-y$ risk of coronary artery disease incidence: the Zutphen Elderly Study. American Journal of Clinical Nutrition 74, 457-463.

Pang D, Allman-Farinelli MA, Wong T, Barnes R \& Kingham KM (1998) Replacement of linoleic acid with alpha-linolenic acid does not alter blood lipids in normolipidaemic men. British Journal of Nutrition 80, 163-167.

Pawlosky RJ, Hibbeln JR, Novotny JA \& Salem N Jr (2001) Physiological compartmental analysis of alpha-linolenic acid metabolism in adult humans. Journal of Lipid Research 42, 1257-1265.

Salem N Jr, Pawlosky R, Wegher B \& Hibbeln J (1999) In vivo conversion of linoleic acid to arachidonic acid in human adults. Prostaglandins, Leukotrienes and Essential Fatty Acids 60, 407-410.

Thies F, Miles EA, Nebe-von-Caron G, Powell JR, Hurst TL, Newsholme EA \& Calder PC (2001a) Influence of dietary supplementation with long-chain n-3 or n-6 polyunsaturated fatty acids on blood inflammatory cell populations and functions and on plasma soluble adhesion molecules in healthy adults. Lipids 36, 1183-1193.

Thies F, Nebe-von-Caron G, Powell JR, Yaqoob P, Newsholme EA \& Calder PC (2001b) Dietary supplementation with 
eicosapentaenoic acid, but not with other long-chain n-3 or n-6 polyunsaturated fatty acids, decreases natural killer cell activity in healthy subjects aged $>55 \mathrm{y}$. American Journal of Clinical Nutrition 73, 539-548.

Thies F, Nebe-von-Caron G, Powell JR, Yaqoob P, Newsholme EA \& Calder PC (2001c) Dietary supplementation with gamma-linolenic acid or fish oil decreases T lymphocyte proliferation in healthy older humans. Journal of Nutrition 131, 1918-1927.

Vermunt SH, Mensink RP, Simonis MM \& Hornstra G (2000) Effects of dietary alpha-linolenic acid on the conversion and oxidation of 13C-alpha-linolenic acid. Lipids 35, 137-142. 